\title{
Characteristics of carotid atherosclerotic plaques of chronic lipid apheresis patients as assessed by In Vivo High-Resolution CMR - a comparative analysis
}

Jochen M Grimm', Konstantin Nikolaou', Andreas Schindler ${ }^{1}$, Reinhard Hettich², Franz Heigl ${ }^{2}$, Clemens C Cyran $^{1}$, Florian Schwarz ${ }^{1}$, Reinhard Klingel ${ }^{3}$, Anna Karpinska ${ }^{5}$, Chun Yuan ${ }^{4}$, Martin Dichgans ${ }^{5}$, Maximilian F Reiser ${ }^{1}$ and Tobias Saam ${ }^{*}$

\begin{abstract}
Background: Components of carotid atherosclerotic plaques can reliably be identified and quantified using high resolution in vivo 3-Tesla CMR. It is suspected that lipid apheresis therapy in addition to lowering serum lipid levels also has an influence on development and progression of atherosclerotic plaques. The purpose of this study was to evaluate the influence of chronic lipid apheresis (LA) on the composition of atherosclerotic carotid plaques.
\end{abstract}

Methods: 32 arteries of 16 patients during chronic LA-therapy with carotid plaques and stenosis of 1-80\% were matched according to degree of stenosis with 32 patients, who had recently suffered an ischemic stroke. Of these patients only the asymptomatic carotid artery was analyzed. All patients underwent black-blood 3 T CMR of the carotids using parallel imaging and dedicated surface coils. Cardiovascular risk factors were recorded. Morphology and composition of carotid plaques were evaluated. For statistical evaluation Fisher's Exact and unpaired $t$-test were used. A p-value $<0.05$ was considered statistically significant.

Results: Patients in the LA-group were younger (63.5 vs. 73.9. years, $p<0.05$ ), had a higher prevalence of hypercholesterolemia and of established coronary heart disease in patients and in first-degree relatives $(p<0.05$, respectively). LA-patients had smaller maximum wall areas $\left(49.7\right.$ vs. $\left.59.6 \mathrm{~mm}^{2}, p<0.05\right)$, showed lower prevalence of lipid cores $(28.1 \%$ vs. $56.3 \%, p<0.05)$ and the lipid content was smaller than in the control group (5.0 vs. $11.6 \%$, $p<0.05)$. Minimum lumen areas and maximum total vessel areas did not differ significantly between both groups.

Conclusion: Results of this study suggest that, despite a severer risk profile for cardiovascular complications in LA-patients, chronic LA is associated with significantly lower lipid content in carotid plaques compared to plaques of patients without LA with similar degrees of stenosis, which is characteristic of clinically stable plaques.

Keywords: Atherosclerosis, Lipid apheresis, Cardiovascular MR, Plaque imaging, Stroke, Cardiovascular disease

\section{Background}

Cardiovascular events, such as myocardial infarction or stroke are the leading cause of death in the western world. The incidence of cardiovascular diseases like ischemic stroke or infarction of heart muscle tissue is well correlated with a number of risk factors. Dyslipidemia is

\footnotetext{
* Correspondence: tobias.saam@med.Imu.de

'Institute for Clinical Radiology, Ludwig-Maximilians-University Hospital

Munich, Pettenkoferstr. 8a, 80336, Munich, Germany

Full list of author information is available at the end of the article
}

one of the most important established risk factors being causally related to cardiovascular events [1].

It has been shown that lowering of pathologically high serum levels of low density lipoprotein cholesterol (LDL-C) either by pharmacotherapy or lipid apheresis therapy significantly reduces cardiovascular morbidity and mortality $[2,3]$. However, how exactly this positive effect is achieved on a pathophysiological level is not completely understood.

One possible explanation is the improvement of rheological properties of the blood after lipid lowering 
therapy [4]. High levels of LDL-C have also been associated with increased inflammatory activity in vessel walls and reduced vasodilatory activity [5,6]. It has also been suspected that lipid lowering therapy in dyslipidemic patients leads to regression of atherosclerotic disease and reduction of the lipid component of atherosclerotic plaques $[7,8]$.

Large vessel atherosclerotic disease is considered responsible for $20-30 \%$ of ischemic strokes [9]. It has been shown that cardiovascular events are correlated not only with the degree of stenosis of the respective vessel but even more so with the properties of the stenosing plaque [10], which promoted the concept of the complicated or vulnerable plaque $[11,12]$. One of the key features of the so-called vulnerable plaques is the large lipid/necrotic core covered by a thin fibrous cap $[11,12]$. This leads to the hypothesis that a decrease in their lipid content stabilizes atherosclerotic plaques.

Lipid apheresis therapy is an effective therapeutic option to lower serum lipid levels in patients with extensive hyperlipidemia, where medication alone is insufficient to reduce serum lipids to target levels [13]. A decrease of the size of atherosclerotic plaques and possible beneficial change of their composition have been reported in dyslipidemic patients under lipid lowering therapy $[7,14,15]$. It has been suspected that dyslipidemia is associated with an increased prevalence of soft plaques [16]. Consequently, a decrease in serum lipid levels should have an influence on prevalence of soft plaques and plaque composition [17] and may lower the fraction of vulnerable plaques among atherosclerotic lesions $[8,18]$. This mechanism should as well be relevant in lipid apheresis possibly caused by a direct lowering effect on the lipid content of atherosclerotic plaques [19]. This might offer an additional explanation for the observed decrease of cardiovascular events during chronic lipid apheresis.

A number of techniques allow for the assessment of atherosclerotic disease, among these extra- and intravascular ultrasound, CT-angiography, conventional angiography and cardiovascular magnetic resonance (CMR) plaque imaging. CMR plaque imaging has become one of the most reliable techniques to assess atherosclerotic plaque morphology and composition in good correlation with histopathology [20,21]. It is non-invasive, involves no ionizing radiation and is well suited to reliably detect and quantify plaque components including the lipid content of atherosclerotic plaques [22].

Existing studies have evaluated the effect of lipid lowering pharmacotherapy on atherosclerotic plaque development using CMR [7] or the effect of lipid apheresis on coronary plaque composition using ultrasound [23]. Other studies evaluated coronary plaque composition in relation to serum lipid levels using computed tomography angiography [17]. One Study evaluated the development of aortic atherosclerotic plaques in relation to serum lipid levels in rabbits using CMR [24]. To our knowledge, no studies have evaluated the effect of chronic lipid apheresis on carotid atherosclerotic plaque composition using CMR plaque imaging in human patients.

The purpose of this prospective comparative cross sectional study was to evaluate the influence of chronic lipid apheresis (LA) on the composition of atherosclerotic carotid plaques using high resolution in vivo 3-Tesla CMR with parallel imaging techniques.

\section{Methods}

\section{Patient selection}

A total of 48 patients were enrolled in this prospective cross sectional, monocentric observational study, which has been approved by the local institutional ethics committee. In the chronic lipid apheresis group we examined 32 arteries of 16 patients who had been treated by chronic lipid apheresis for more than 5 years (7 patients by heparin mediated plasma precipitation in acidic milieu (HELP (C), 7 patients by temperature optimized double-filtration plasmapheresis (Lipidfiltration (), 2 patients by polyacrylat full blood adsorption (DALI (C)). Indication for chronic lipid apheresis was severe therapy refractory hypercholesterolemia $(\mathrm{N}=13)$ or isolated $\mathrm{Lp}(\mathrm{a})$ hyperlipoproteinemia with progressive vascular disease $(\mathrm{N}=3)$. Lipid apheresis treatment had been performed in intervals between 3 and 14 days. In the control group we included 32 consecutive patients matched for the degree of vascular stenosis, who had recently suffered an ischemic stroke. Of these patients only the asymptomatic artery contralateral to the ischemic stroke was included. The majority of patients from the lipid apheresis group ( 9 out of 16, 56\%) and control group (20 out of 32, $63 \%)$ received lipid lowering medication at the time of examination. Manifest coronary artery disease and cardiovascular risk factors including serum lipid levels, arterial hypertension, active or past nicotine abuse, diabetes mellitus, family history of coronary artery disease and body mass index (BMI) were recorded. Values for LDL and Lp (a) for the lipid apheresis group are given as mean levels calculated as proposed by Kroon et al. [25]. Other serum lipid levels for the lipid apheresis group are given as average of measurements before and after apheresis therapy. Serum lipid levels for the control group were measured at the time of the CMR examination performed at time of admission due to acute ischemic stroke.

\section{MR imaging protocol}

All subjects underwent a carotid black-blood high resolution CMR at 3.0-Tesla on a Siemens Verio Scanner (Siemens AG, Erlangen, Germany) using surface coils (Machnet, Elde, Netherlands) and a previously published multi-sequence protocol [26] (time-of-flight MR angiography (TOF), axial fat suppressed pre- and post- contrast 
black-blood T1-, PD- and T2- weighted sequences; best in-plane resolution $0.5 \times 0.5 \mathrm{~mm}^{2}$ ). Parallel imaging based on the generalized autocalibrating partially parallel acquisition (GRAPPA) algorithm was used for all sequences with a parallel acquisition technique (PAT) acceleration factor of 2. Total imaging time was 17:43 minutes. Gadobutrol (Gadovist ${ }^{\circledR}$, Bayer Schering, Leverkusen, Germany) of $0.1 \mathrm{mmol} / \mathrm{kg}(0.1 \mathrm{ml} / \mathrm{kg})$ was given at a rate of $3 \mathrm{ml} / \mathrm{s}$. Post-contrast T1w imaging was performed approximately 5 minutes after intravenous injection of the contrast agent. Each scan covered $30 \mathrm{~mm}$ ( $2 \mathrm{~mm}$ slice thickness $\times$ 15 matched images across the 5 sequences).

\section{Image analysis}

An image-quality rating (4-point scale, 1 = non-diagnostic, 2 = poor, 3 = good, 4 = excellent) was assigned to all MR images. MR data were then independently reviewed by two experienced radiologists (T.S. and J.G.) who where blinded regarding the clinical status of the patient. In case of discrepancy, a consensus decision was made. Atherosclerotic plaques in the carotid arteries were recorded and classified according to the modified criteria of the American Heart Association [27]. For definition of a complicated AHA-LT6 plaque, at least one of the following three criteria was required: fibrous cap rupture, intraplaque hemorrhage, juxtaluminal hemorrhage/mural thrombus. Area measurements of the lumen, outer wall, and tissue components were obtained using a customdesigned image analysis tool CASCADE (University of Washington, Seattle, USA). Tissue components (lipid-rich necrotic core, calcification, hemorrhage) were identified based on previously published criteria [20]. The "total vessel area" included the lumen and wall areas. The normalized wall index (NWI) was calculated by dividing the wall area by the total vessel area.

\section{Statistical analysis}

For statistical comparison of the patients with and without lipid apheresis therapy, the Mann-Whitney test with correction for ties was used for variables describing frequencies of plaque features, which had skewed distribution. For continuous variables, the Student unpaired $t$-test with equal or unequal variances (as appropriate) was used. To compare clinical demographics between both groups, Fisher's exact test was used for categorical variables, and the unpaired $t$-test was used for continuous variables. Categorical variables are presented as absolute and relative frequencies, while continuous variables are presented as mean and standard deviation. Tissue components were calculated as percentages of the vessel wall. Analyses were carried out in SPSS for Windows (version 14.0, IBM, Chicago, IL). A p-value of $<0.05$ was considered as statistically significant.

\section{Results}

\section{Clinical data}

Patients in the lipid apheresis group were younger (63.5 vs. 73.9 years, $\mathrm{p}<0.05)$ and had a higher prevalence of hypercholesterolemia (100\% vs. $53 \%)$, coronary artery disease and/or family history of coronary artery disease (100\% vs. $34 \%$ ) (all p-values <0.05). Total cholesterol (148.8 vs. $200.8 \mathrm{mg} / \mathrm{dl}$ ) and LDL (94.7 vs. $128.3 \mathrm{mg} / \mathrm{dl}$ ) averaged as described above - were significantly lower in the lipid apheresis group than in the control group $(\mathrm{p}<0.001)$. Consequently, LDL/HDL was also lower in the lipid apheresis group ( 2.0 vs. $2.8 ; \mathrm{p}<0.01)$. No further statistically significant differences in clinical data were found [Table 1].

\section{Imaging data \\ Image quality}

48 out of 50 scans (96.0\%) had a sufficient image quality (image quality $\geq 2$ ), with an average image quality rating of 3.6 for the lipid apheresis patients and 3.4 for the control group $(\mathrm{p}=\mathrm{ns})$. The two excluded scans were scans of the control group. All data are given for 16 lipid apheresis patients and 32 patients of the control group. Imaging examples are given [Figures 1 and 2].

\section{Qualitative and quantitative plaque imaging characteristics}

Patients in the lipid apheresis group showed a lower prevalence of lipid cores compared to the control subjects ( 28.1 vs. $56.3 \%$; $\mathrm{p}<0.05$ ). The maximum lipid content as percentage of the vessel wall was also smaller in the lipid apheresis group (5.0 vs. $11.6 \%, \mathrm{p}<0.05)$. There was a tendency towards larger minimum lumen areas in the lipid apheresis group compared to the control group, however, both parameters did not differ significantly between the two groups ( 15.9 vs. $\left.13.0 \mathrm{~mm}^{2}, \mathrm{p}=\mathrm{ns}\right)$. The normalized wall index and the maximum wall area were smaller in the lipid apheresis group than in the control group (0.61 vs. $0.72 ; \mathrm{p}<0.001$ and 49.7 vs. $59.6 \mathrm{~mm}^{2}$; $\mathrm{p}<0.05)$. Prevalence of plaque calcifications and calcification content did not differ between both groups. Intraplaque hemorrhage prevalence and hemorrhage content did not differ significantly between both groups (9.4\% vs. $15.6 \%$ and 2.1 vs. $2.3 \%$; $\mathrm{p}=\mathrm{ns}$ ), although there was a tendency towards a higher prevalence of hemorrhages in the control group [Table 2].

\section{AHA lesion type distribution}

AHA lesion type IV/V, which is characterized by the presence of a lipid core covered by a fibrous cap, was found significantly more often in the control group compared to the lipid apheresis group ( $38 \%$ vs. $13 \%$; $\mathrm{p}<0.05$ ). No other significant differences were found although there was a tendency towards a higher prevalence of calcified AHA type VII lesions in the lipid apheresis group 
Table 1 Cinical data

\begin{tabular}{|c|c|c|c|}
\hline Parameter & $\begin{array}{l}\text { Lipid apheresis group } \\
\text { ( } n=16 \text { patients) }\end{array}$ & $\begin{array}{l}\text { Control group } \\
\text { ( } n=32 \text { patients) }\end{array}$ & $p$-value* \\
\hline Age \pm 1 SD [years] & $63.5 \pm 9,1$ & $73.9 \pm 9.2$ & $<0.05$ \\
\hline Male [\%] & $68,8 \%$ & $84.4 \%$ & ns \\
\hline Body Mass Index & $26.5 \pm 4.4$ & $25.1 \pm 5.3$ & ns \\
\hline Established CAD [\%] & $100 \%$ & $21.9 \%$ & $<0.001$ \\
\hline \multicolumn{4}{|l|}{ Risk Factors } \\
\hline Hypercholesterolemia [\%] & $100 \%$ & $53.3 \%$ & 0.008 \\
\hline Arterial Hypertension, [\%] & $75 \%$ & $68.8 \%$ & ns \\
\hline Active Nicotine Abuse [\%] & $6.3 \%$ & $28.1 \%$ & ns \\
\hline Former Nicotine Abuse [\%] & $50 \%$ & $40.7 \%$ & ns \\
\hline Diabetes Mellitus [\%] & $18.8 \%$ & $21.9 \%$ & ns \\
\hline Family History of CAD or CVD [\%] & $68.8 \%$ & $34.4 \%$ & 0.03 \\
\hline \multicolumn{4}{|l|}{ Serum Lipids } \\
\hline Total Cholesterol [mg/dl] & $148.8 \pm 37.7$ & $200.8 \pm 47.1$ & $<0.001$ \\
\hline Triglycerides $[\mathrm{mg} / \mathrm{dll}]$ & $139.1 \pm 93.9$ & $142.0 \pm 73.5$ & ns \\
\hline $\mathrm{LDL}[\mathrm{mg} / \mathrm{dl}]$ & $94.7 \pm 28.9$ & $128.3 \pm 35.8$ & $<0.001$ \\
\hline VLDL [mg/dl] & $18.6 \pm 7.9$ & $22.4 \pm 10.4$ & ns \\
\hline $\mathrm{HDL}[\mathrm{mg} / \mathrm{dl}]$ & $49.3 \pm 12.7$ & $48.2 \pm 9.2$ & ns \\
\hline $\mathrm{LDL} / \mathrm{HDL}$ & $2.0 \pm 1.0$ & $2.8 \pm 0.8$ & 0.008 \\
\hline Lipoprotein (a) [mg/dl] & $59.9 \pm 66.6$ & $47.4 \pm 49.4$ & ns \\
\hline
\end{tabular}

Two-sided Fisher's Exact Test or unpaired t-test; SD=Standard Deviation; CAD=Coronary Artery Disease; CVD=Cerebrovascular Disease (TIA or Stroke). Values for LDL and Lp (a) for the lipid apheresis group are given as mean levels calculated as proposed by Kroon et al. [25]. Other serum lipid levels for the lipid apheresis group are given as average of measurements before and after apheresis therapy.
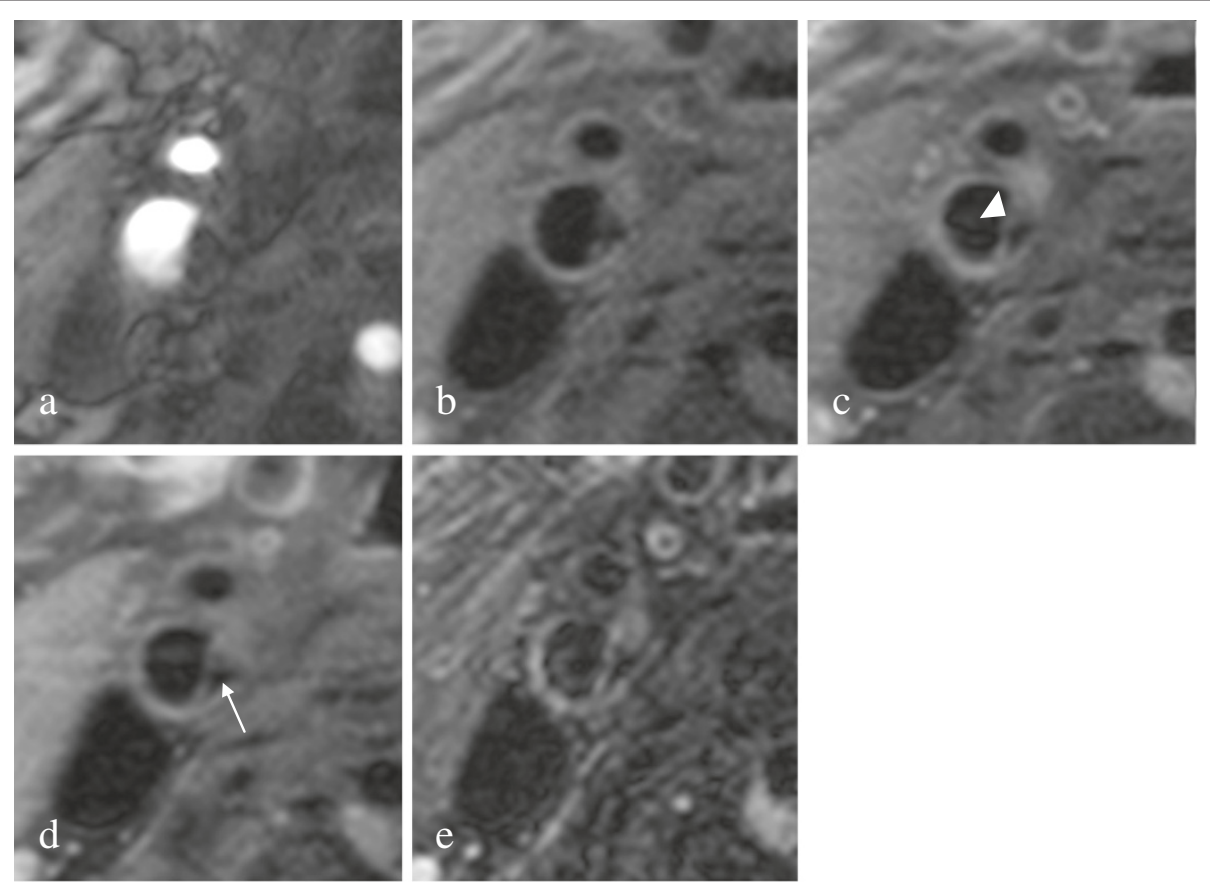

Figure 1 Imaging example Lipid Apheresis Group. shows axial TOF (a), T1w without (b) and with (c) contrast agent, PD (d) and T2w (e) MRimages of an American Heart Association Type II plaque in the right internal carotid artery of a patient from the lipid apheresis group. This plaque is mainly calcified (arrow); no lipid necrotic core can be seen. The fibrous cap appears intact (arrowhead). 

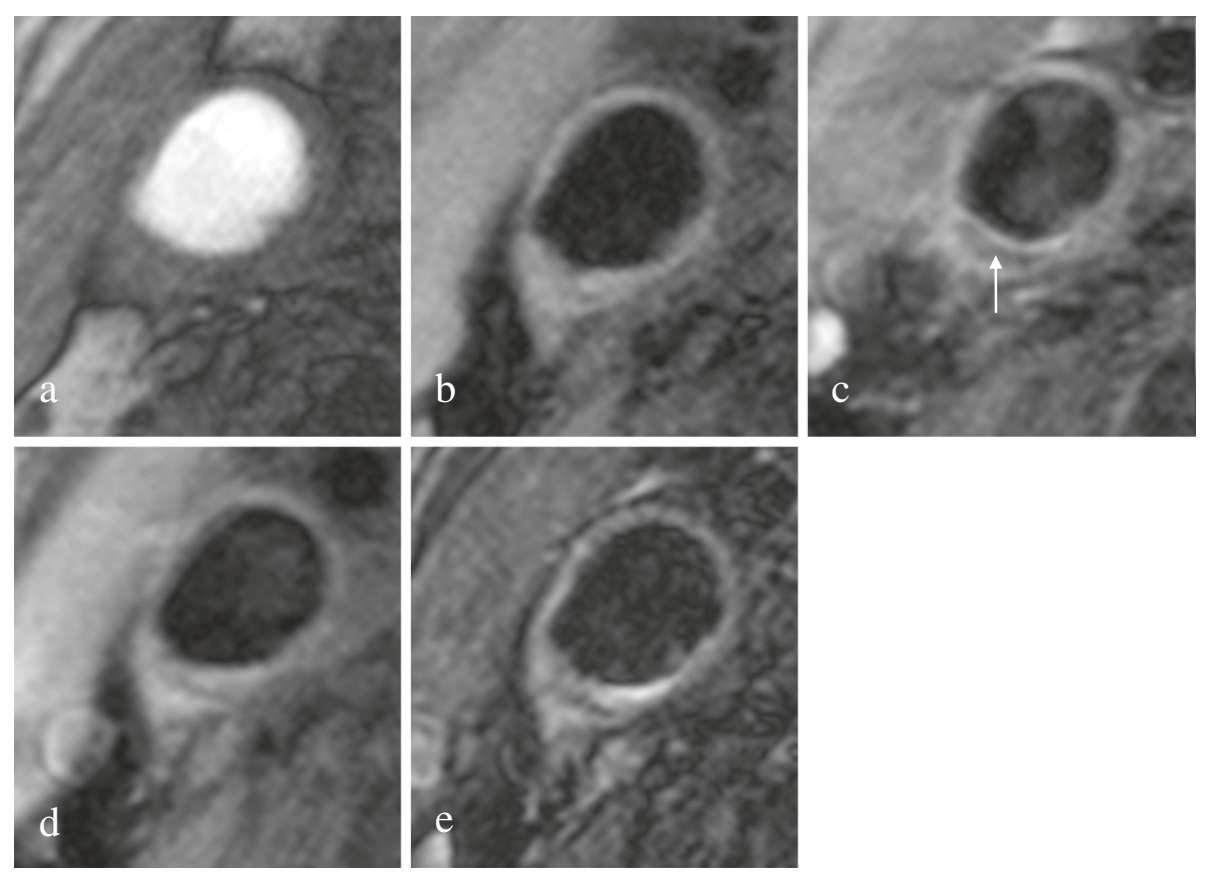

Figure 2 Imaging Example Control Group. shows an American Heart Association type $V$ plaque at the bifurcation in the right internal carotid artery of a patient from the control group in axial TOF (a), T1w without (b) and with (c) contrast agent, PDw (d) and T2w (e) MR-images. The plaque consists mainly of a necrotic lipid core without contrast enhancement (arrow) and no relevant calcification.

compared to the control group ( $38 \%$ vs. $19 \% ; \mathrm{p}=\mathrm{ns})$ [Figure 3].

\section{Discussion}

This cross-sectional study showed that patients who had been treated by chronic lipid apheresis therapy for more than 5 years had a significantly lower prevalence of lipid-rich plaques and lower plaque lipid content compared to asymptomatic arteries with similar degrees of stenosis. Patients on chronic lipid apheresis therapy had a lower wall area and a tendency towards more calcified plaques compared to the control group. Accordingly, total cholesterol, LDL and LDL/HDL were lower in the lipid apheresis group suggesting an influence of these parameters on plaque characteristics. Plaques of patients during chronic lipid apheresis showed characteristics of more stable plaques compared to the control group. This study also demonstrated significant differences in AHA lesion type distribution. AHA lesion type IV/V, characterized by a lipid core covered by a fibrous cap was significantly more common in the control group, whereas the relatively stable calcified AHA lesion type VII had a higher prevalence in the lipid apheresis group. In summary, these findings suggest a beneficial influence of lipid apheresis therapy on plaque composition despite the significantly severer risk profile in the LA-group considering the higher prevalence of hypercholesterolemia and positive family history of cardiovascular disease in LA-patients.

This result is in accordance with previously published studies, which suspected a decrease in the lipid component of atherosclerotic plaques of patients under lipid lowering therapy $[17,19]$. Underhill et al. [23] showed in a double-blind trial using 1.5-T CMR to image carotid atherosclerotic plaques at baseline and after 24 months of rosuvastatin treatment a significant reduction in lipid rich necrotic core, whereas the overall plaque burden remained unchanged over the course of 2 years of treatment. The authors concluded that statin therapy may have a beneficial effect on plaque volume and composition, as assessed by non-invasive CMR. Results of this study support the proposition that serum lipid levels correlate with lipid content of atherosclerotic lesions. Since lipid rich plaques seem to be a more vulnerable subgroup of atherosclerotic lesions, a decrease of their lipid component might help explain the decrease in cardiovascular incidents [8].

The minimal lumen area as an indirect measurement of stenosis was similar in both groups. We found smaller maximum wall areas in the lipid apheresis group and smaller normalized wall indices, suggesting a lower plaque burden in this group of patients. This might in part be explained by previous studies which have not found a significant increase in vessel lumen area of 
Table 2 MR-Imaging data

\begin{tabular}{|c|c|c|c|}
\hline & $\begin{array}{l}\text { Lipid apheresis group } \\
\text { ( } n=32 \text { arteries) }\end{array}$ & $\begin{array}{l}\text { Control group } \\
(\mathrm{n}=32 \text { arteries })\end{array}$ & p-value* \\
\hline Lumen Area & $15.9 \pm 6.3$ & $13.0 \pm 7.6$ & ns \\
\hline \multicolumn{4}{|l|}{$\left[\mathrm{mm}^{2}\right.$, Minimum] } \\
\hline Wall Area & $49.7 \pm 11.8$ & $59.6 \pm 21.6$ & 0.03 \\
\hline \multicolumn{4}{|l|}{$\left[\mathrm{mm}^{2}\right.$, Maximum] } \\
\hline Normalized Wall Index & $0.61 \pm 0.09$ & $0.72 \pm 0.12$ & $<0.001$ \\
\hline \multicolumn{4}{|l|}{ [Maximum] } \\
\hline Outer Wall Area & $105.4 \pm 28.3$ & $102.0 \pm 35.4$ & ns \\
\hline \multicolumn{4}{|l|}{$\left[\mathrm{mm}^{2}\right.$, Maximum] } \\
\hline \multicolumn{4}{|c|}{ Plaque Components (quantitative) } \\
\hline Necrotic Lipid Core & $5.0 \pm 9.4$ & $11.6 \pm 13.4$ & 0.025 \\
\hline \multicolumn{4}{|l|}{ [\% of Vessel Wall, Maximum] } \\
\hline Intraplaque Hemorrhage & $2.1 \pm 8.8$ & $2.3 \pm 6.8$ & ns \\
\hline \multicolumn{4}{|l|}{ [\% of Vessel Wall, Maximum] } \\
\hline Calcifications & $4.8 \pm 6.0$ & $5.3 \pm 6.1$ & ns \\
\hline \multicolumn{4}{|l|}{ [\% of Vessel Wall, Maximum] } \\
\hline \multicolumn{4}{|c|}{ Prevalence of Plaque Components } \\
\hline Necrotic Lipid Core [\%] & 28.1 & 56.3 & 0.025 \\
\hline Intraplaque Hemorrhage [\%] & 9.4 & 15.6 & ns \\
\hline Calcifications [\%] & 53.1 & 56.3 & ns \\
\hline \multicolumn{4}{|c|}{ American Heart Association (AHA) Lesion Type } \\
\hline Type I [\%] & 15.6 & 12.5 & ns \\
\hline Type III [\%] & 25.0 & 15.6 & ns \\
\hline Type IVN [\%] & 13.0 & 37.5 & 0.023 \\
\hline Type VI [\%] & 9.4 & 15.6 & ns \\
\hline Type VII [\%] & 37.5 & 18.8 & ns \\
\hline Type VIII [\%] & 0 & 0 & ns \\
\hline
\end{tabular}

* Unpaired t-test; SD=Standard Deviation.

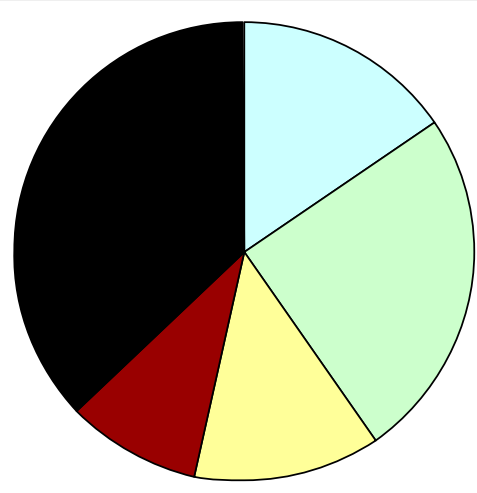

Lipid Apheresis Group ( $\mathrm{N}=32$ arteries)

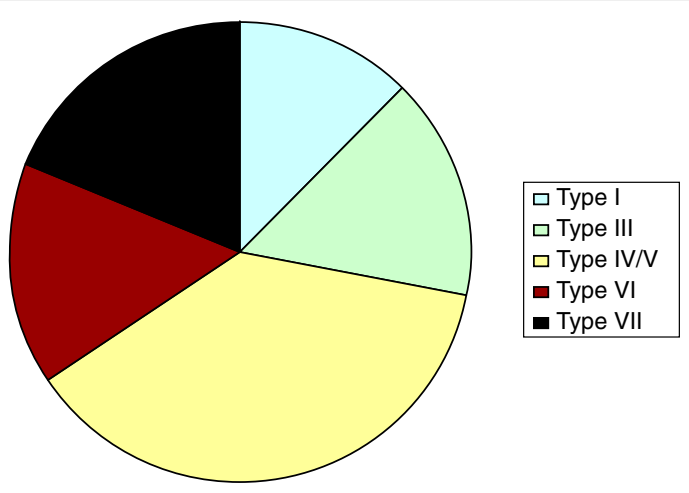

Control Group ( $\mathrm{N}=32$ arteries)

Figure 3 AHA Lesion Type Distribution. Visualizes the distribution of American Heart Association (AHA) lesion Types in the Lipid Apheresis and the Control Group. Note the higher prevalence of lipid rich type IVN plaques in the Control Group. There also was a tendency toward higher prevalence of calcified Type VII plaques in the Lipid Apheresis Group. 
treated dyslipidemic patients over time while showing a significant decrease in vessel wall area [7].

Although the number of patients enrolled was relatively small, significant differences in atherosclerotic disease between lipid apheresis patients and the control group were identified. This study suggests that CMR is a powerful tool to noninvasively evaluate atherosclerotic disease systematically across different groups of patients and populations.

This study achieved diagnostic image quality in 48 out of 50 scans, demonstrating that this protocol can be successfully used in a clinical setting. This drop-out rate of only $4 \%$ is substantially lower than the drop-out rates reported in recent 1.5 T MR studies, in which up to $33 \%$ of all patients were excluded due to insufficient image quality $[28,29]$.

\section{Limitations}

Although this study shows significant differences in plaque morphology and lesion type distribution between the two groups, the number of patients in this study is relatively small, and a larger study performed in multiple centers is necessary to confirm these preliminary findings. Furthermore, the cross-sectional design of the study allows by definition only a snapshot of plaque burden and composition at one single time point and no definite conclusions can be drawn on the evolution of plaque composition over time. Only CMR examinations with at least average image quality were considered for the review, resulting in exclusion of two arteries from analysis. However, this exclusion rate is lower than in previously published studies $[28,29]$ and should not have hampered the results. These results were based on a single imaging modality: CMR. Information gathered from other well-known imaging techniques, notably measurements of calcifications from computed tomography, might further illuminate the differences. However, CMR is generally considered best suited to identify and quantify atherosclerotic plaque components in the carotid arteries, shows good correlation with histology for quantitative measurements, and does not use ionizing radiation. Given these advantages and the comparative complexity of a multi-modality study, sole use of CMR was the logical choice for this study.

\section{Conclusion}

Findings of this study suggest that chronic lipid apheresis therapy is associated with lower cholesterol, LDL and LDL/HDL as well as a markedly decreased lipid content in atherosclerotic carotid plaques as assessed by in vivo CMR as compared to a control group with similar degree of arterial stenosis. These findings suggest an influence of cholesterol, LDL and LDL/HDL on plaque characteristics as well as a stabilizing effect of chronic lipid apheresis therapy on carotid plaque composition and might explain the observed beneficial influence of lipid apheresis therapy on clinical outcome. In the future, prospective longitudinal studies are needed to confirm these promising results and to better assess individual plaque development over time during chronic lipid apheresis.

\section{Competing interests}

The study was sponsored by Diamed.

\section{Authors' contributions}

JMG participated in data acquisition, statistical evaluation, drafted and revised the manuscript. KN participated in designing the study and revising the manuscript. AS participated in patient and data acquisition. $\mathrm{RH}$ participated in designing the study and revising the manuscript. FH participated in designing the study and revising the manuscript. CCC participated in patient and data acquisition. FS participated in patient and data acquisition. RK participated in designing the study and revising the manuscript. AK participated in evaluation of the data. CY participated in evaluating the data and revising the manuscript. MD participated in designing the study, evaluating the data and revising the manuscript. MFR participated in designing the study and revising the manuscript. TS participated in designing the study, acquiring and evaluating the data, and revising the manuscript. All authors read and approved the final manuscript.

\section{Author details}

${ }^{1}$ Institute for Clinical Radiology, Ludwig-Maximilians-University Hospital Munich, Pettenkoferstr. 8a, 80336, Munich, Germany. ${ }^{2}$ Medizinisches Versorgungszentrum Kempten-Allgäu, Kempten-Allgäu, Germany. ${ }^{3}$ Apheresis Research Institute, Cologne, Germany. ${ }^{4}$ Department of Radiology, University of Washington School of Medicine, Seattle, USA. Institute for Stroke and Dementia Research, Ludwig-Maximilians-University Hospital Munich, Munich, Germany.

Received: 3 July 2012 Accepted: 16 November 2012

Published: 29 November 2012

\section{References}

1. Goldstein LB, Bushnell CD, Adams RJ, Appel LJ, Braun LT, Chaturvedi S, Creager MA, Culebras A, Eckel RH, Hart RG, et al.: Guidelines for the primary prevention of stroke: a guideline for healthcare professionals from the American Heart Association/American Stroke Association. Stroke 2011, 42:517-84.

2. Barter PJ: Coronary plaque regression: role of low density lipoproteinapheresis. J Am Coll Cardiol 2002, 40:228-30.

3. Mabuchi H, Koizumi J, Shimizu M, Kajinami K, Miyamoto S, Ueda K, Takegoshi T: Long-term efficacy of low-density lipoprotein apheresis on coronary heart disease in familial hypercholesterolemia. Hokuriku-FHLDL-Apheresis Study Group. Am J Cardiol 1998, 82:1489-95.

4. Schuff-Werner P, Schutz E, Seyde WC, Eisenhauer T, Janning G, Armstrong W, Seidel D: Improved haemorheology associated with a reduction in plasma fibrinogen and LDL in patients being treated by heparin-induced extracorporeal LDL precipitation (HELP). Eur J Clin Invest 1989, 19:30-7.

5. Mangge H, Almer G, Truschnig-Wilders M, Schmidt A, Gasser R, Fuchs D: Inflammation, adiponectin, obesity and cardiovascular risk. Curr Med Chem 2010, 17:4511-20.

6. Mellwig KP, van Buuren F, Schmidt HK, Wielepp P, Burchert W, Horstkotte D: Improved coronary vasodilatatory capacity by H.E.L.P. apheresis: comparing initial and chronic treatment. Ther Apher Dial 2006, 10:510-7.

7. Corti R, Fayad ZA, Fuster V, Worthley SG, Helft G, Chesebro J, Mercuri M, Badimon JJ: Effects of lipid-lowering by simvastatin on human atherosclerotic lesions: a longitudinal study by high-resolution, noninvasive magnetic resonance imaging. Circulation 2001, 104:249-52.

8. Brown $B G$, Zhao XQ, Sacco DE, Albers JJ: Lipid lowering and plaque regression. New insights into prevention of plaque disruption and clinical events in coronary disease. Circulation 1993, 87:1781-91.

9. Albers GW, Amarenco P, Easton JD, Sacco RL, Teal P: Antithrombotic and thrombolytic therapy for ischemic stroke: American College of Chest Physicians Evidence-Based Clinical Practice Guidelines (8th Edition). Chest 2008, 133:630S-69. 
10. Yuan C, Mitsumori LM, Beach KW, Maravilla KR: Carotid atherosclerotic plaque: noninvasive MR characterization and identification of vulnerable lesions. Radiology 2001, 221:285-99.

11. Naghavi M, Libby P, Falk E, Casscells SW, Litovsky S, Rumberger J, Badimon JJ, Stefanadis C, Moreno P, Pasterkamp G, et al.: From vulnerable plaque to vulnerable patient: a call for new definitions and risk assessment strategies: Part II. Circulation 2003, 108:1772-8.

12. Naghavi M, Libby P, Falk E, Casscells SW, Litovsky S, Rumberger J, Badimon $\mathrm{JJ}$, Stefanadis C, Moreno P, Pasterkamp G, et al.: From vulnerable plaque to vulnerable patient: a call for new definitions and risk assessment strategies: Part I. Circulation 2003, 108:1664-72.

13. Thompson GR: Recommendations for the use of LDL apheresis. Atherosclerosis 2008, 198:247-55.

14. Underhill HR, Yuan C, Zhao XQ, Kraiss LW, Parker DL, Saam T, Chu B, Takaya $\mathrm{N}$, Liu F, Polissar NL, et al.: Effect of rosuvastatin therapy on carotid plaque morphology and composition in moderately hypercholesterolemic patients: a high-resolution magnetic resonance imaging trial. Am Heart J 2008, 155:584 e1-584 e8.

15. Zhao XQ, Yuan C, Hatsukami TS, Frechette EH, Kang XJ, Maravilla KR, Brown $B G$ : Effects of prolonged intensive lipid-lowering therapy on the characteristics of carotid atherosclerotic plaques in vivo by MRI: a case-control study. Arterioscler Thromb Vasc Biol 2001, 21:1623-9.

16. Makino K, Yoshitama T, Kanda S, Takasawa Y, Yamada T, Itaya H, Lee T, Saeki F, Nakamura M, Sugi K: Relation of coronary plaque composition determined by 64 -slice multidetector computed tomography in patients with suspected coronary heart disease. Am J Cardiol 2011, 107:1624-9.

17. Cheng VY, Wolak A, Gutstein A, Gransar H, Wong ND, Dey D, Thomson LE, Hayes SW, Friedman JD, Slomka PJ, Berman DS: Low-density lipoprotein and noncalcified coronary plaque composition in patients with newly diagnosed coronary artery disease on computed tomographic angiography. Am J Cardiol 2010, 105:761-6.

18. Brown $B G$, Zhao $X Q$, Sacco DE, Albers Jj: Atherosclerosis regression, plaque disruption, and cardiovascular events: a rationale for lipid lowering in coronary artery disease. Annu Rev Med 1993, 44:365-76.

19. Schuff-Werner P, Schettler V: [Plaque stabilization by LDL apheresis?]. Herz 1999, 24:57-61.

20. Saam T, Hatsukami TS, Takaya N, Chu B, Underhill H, Kerwin WS, Cai J, Ferguson MS, Yuan C: The vulnerable, or high-risk, atherosclerotic plaque: noninvasive MR imaging for characterization and assessment. Radiology 2007, 244:64-77.

21. Cai J, Hatsukami TS, Ferguson MS, Kerwin WS, Saam T, Chu B, Takaya N, Polissar NL, Yuan C: In vivo quantitative measurement of intact fibrous cap and lipid-rich necrotic core size in atherosclerotic carotid plaque: comparison of high-resolution, contrast-enhanced magnetic resonance imaging and histology. Circulation 2005, 112:3437-44.

22. Saam T, Ferguson MS, Yarnykh VL, Takaya N, Xu D, Polissar NL, Hatsukami TS, Yuan C: Quantitative evaluation of carotid plaque composition by in vivo MRI. Arterioscler Thromb Vasc Biol 2005, 25:234-9.

23. Matsuzaki M, Hiramori K, Imaizumi T, Kitabatake A, Hishida H, Nomura M, Fujii T, Sakuma I, Fukami K, Honda T, et al.: Intravascular ultrasound evaluation of coronary plaque regression by low density lipoproteinapheresis in familial hypercholesterolemia: the Low Density LipoproteinApheresis Coronary Morphology and Reserve Trial (LACMART). J Am Coll Cardiol 2002, 40:220-7.

24. Helft G, Worthley SG, Fuster V, Fayad ZA, Zaman AG, Corti R, Fallon JT, Badimon JJ: Progression and regression of atherosclerotic lesions: monitoring with serial noninvasive magnetic resonance imaging. Circulation 2002, 105:993-8.

25. Kroon AA, van't Hof MA, Demacker PN, Stalenhoef AF: The rebound of lipoproteins after LDL-apheresis. Kinetics and estimation of mean lipoprotein levels. Atherosclerosis 2000, 152:519-26.

26. Saam T, Raya JG, Cyran CC, Bochmann K, Meimarakis G, Dietrich O, Clevert DA, Frey U, Yuan C, Hatsukami TS, et al.: High resolution carotid blackblood 3 T MR with parallel imaging and dedicated 4-channel surface coils. J Cardiovasc Magn Reson 2009, 11:41.

27. Cai JM, Hatsukami TS, Ferguson MS, Small R, Polissar NL, Yuan C Classification of human carotid atherosclerotic lesions with in vivo multicontrast magnetic resonance imaging. Circulation 2002, 106:1368-73.

28. Boussel L, Arora S, Rapp J, Rutt B, Huston J, Parker D, Yuan C, Bassiouny H, Saloner D: Atherosclerotic plaque progression in carotid arteries: monitoring with high-spatial-resolution MR imaging-multicenter trial. Radiology 2009, 252:789-96.

29. Parmar JP, Rogers WJ, Mugler JP 3rd, Baskurt E, Altes TA, Nandalur KR, Stukenborg GJ, Phillips CD, Hagspiel KD, Matsumoto AH, et al.: Magnetic resonance imaging of carotid atherosclerotic plaque in clinically suspected acute transient ischemic attack and acute ischemic stroke. Circulation 2010, 122:2031-8.

doi:10.1186/1532-429X-14-80

Cite this article as: Grimm et al:: Characteristics of carotid atherosclerotic plaques of chronic lipid apheresis patients as assessed by In Vivo HighResolution CMR - a comparative analysis. Journal of Cardiovascular Magnetic Resonance 2012 14:80.

\section{Submit your next manuscript to BioMed Central and take full advantage of:}

- Convenient online submission

- Thorough peer review

- No space constraints or color figure charges

- Immediate publication on acceptance

- Inclusion in PubMed, CAS, Scopus and Google Scholar

- Research which is freely available for redistribution 